\title{
Mythos
}

MYTHOS

Rivista di Storia delle Religioni

$10 \mid 2016$

Varia

\section{Attilio Mastrocinque, Bona Dea and the Cults of Roman Women}

Rosalia Marino

\section{(2) OpenEdition}

Edizione digitale

URL: http://journals.openedition.org/mythos/547

DOI: $10.4000 /$ mythos. 547

ISSN: 2037-7746

\section{Editore}

Salvatore Sciascia Editore

\section{Edizione cartacea}

Data di pubblicazione: 1 dicembre 2016

Paginazione: 252-254

ISBN: 978-88-8241-476-4

ISSN: 1972-2516

\section{Notizia bibliografica digitale}

Rosalia Marino, «Attilio Mastrocinque, Bona Dea and the Cults of Roman Women », Mythos [Online], 10 | 2016, online dal 24 septembre 2019, consultato il 25 septembre 2020. URL : http://

journals.openedition.org/mythos/547 ; DOI : https://doi.org/10.4000/mythos.547

Mythos 


\section{Attilio Mastrocinque}

\section{Bona Dea and the Cults of Roman Women}

Franz Steiner (Potsdamer Altertumswissenschaftliche Beiträge 49), Stuttgart 2014, pp. 209, ISBN 978-3-515-10752-5, € 52

Rosalia Marino - Università degli Studi di Palermo - rosalia.marino@unipa.it

Inserito nella prestigiosa serie Potsdamer Altertumswissenschaftliche Beiträge, il saggio di Attilio Mastrocinque affronta, con coerenza di argomentazioni, un tema ricco di implicazioni culturali, che dal piano storico-religioso trascorre a quello politico e socio-antropologico. L'itinerario seguito dallo studioso per rintracciare gli incunaboli del mito di Bona Dea e delinearne il radicamento sul territorio gli consente - da una prospettiva diacronica e, insieme, sincronica - di portare in superficie la consistenza e la resistenza di forme cultuali a salvaguardia dei valori fondanti della società romana, il matrimonio, cioè, e la fertilità.

L'articolazione della complessa materia studiata con sguardo analitico sulle svariate tipologie di fonti disponibili, evidenzia l'intreccio fra storia della mentalità e storia politica, che, non di rado, finiva per tradursi in tensione dialettica. E ciò in coerenza con il carattere sociale della religione romana fatta, com'è noto, di atti di culto e praticata dal singolo individuo in quanto membro di una comunità. II sentimento che dominava la religione romana riguardava, infatti, essenzialmente la volontà di garantire la salvezza della res publica tramite la scrupolosa osservanza della tradizione, funzionale alla garanzia di successo della "pia" Roma ${ }^{1}$.

Nel quadro frastagliato di pratiche e relazioni prematrimoniali tra future spose e dèi, all'insegna di forme di approccio consenzienti (?), rappresentate nella tradizione come nobilitanti ierogamie, grande

1 Sull'errore "religioso" che scaturisce dall'infrazione dei precetti del culto da parte dell'individuo, si veda particolarmente J. Scheid, La religione a Roma, Bari 1983, 8 ss. centralità viene data dallo studioso ad uno dei più importanti initiatory compounds: la Bona Dea, Fauno e i rituali bacchici. In chiave comparativa vengono esplorati i culti presenti nel Lazio, in Etruria e in altre aree italiche, di cui si evidenziano le linee di continuità nei medesimi ambiti cultuali, funzionali alla protezione divina per le donne nel corso delle fasi vitali successive al matrimonio (gravidanza, parto, allattamento). La scelta, poi, di privilegiare, nell'ampio spettro di divinità garanti delle suddette funzioni, l'incisività del culto di Bona Dea si è rivelata di grande significato politico-culturale grazie alle scansioni strutturali che, sub specie di pattern di grande respiro creano un dialogo ininterrotto di Mastrocinque con le fonti capillarmente interrogate.

E così trovano diritto di cittadinanza nessi polifonici di protagonismi divini che esprimono simbolicamente un "attivismo sessuale" che mutua per lo più dal mondo dionisiaco che sembra storicamente chiamato ad assorbire la forza di contrasti ideologici, modalità ricorrenti e finalità coincidenti nel culto di Bona Dea (vd. p. 15) con risalenze mitiche che affondano le radici in un ambito geoculturale di vaste proporzioni.

$\mathrm{Ma}$, di là da ipotetiche ascendenze, l'impressione di una sudditanza della donna nell'approccio amoroso sembra far capolino dalla tradizione che si spinge a "normalizzare" forme evidenti di incesto le quali, senza intaccare il valore sociale riconosciuto con una certa enfasi alle attività riproduttive, creano, attraverso la concessione di feste per sole donne e a guida matronale, una sorta di protagonismo parallelo surrettiziamente teorizzato da accorte strategie storiografiche. Queste, infatti, in momenti di gravi 
fratture politiche, enfatizzavano la funzione di collante sociale della donna riconoscendone il ruolo di soggetto attivo nel corpo dello Stato. Ma gli ingredienti dei riti propedeutici, come quello di Bona Dea, al gradimento del rapporto sessuale per le fanciulle, continuavano a ribadire - è il mio punto di vista - un primato al maschile attraverso il richiamo a simboli fallocratici o a modalità orgiastiche di ambito dionisiaco, descritti in più momenti e con ricchezza di particolari da Mastrocinque.

Paradigmatica, di questo nucleo di idee, la vicenda relativa alla profanazione, da parte di Clodio, della festa di Bona Dea, celebrata nel dicembre del 62, in casa di Cesare, allora pretore e pontefice massimo. La narrazione dello scandalo da prospettive divaricate, che riflettono il livello di crisi della res publica romana, è la spia più evidente del rapporto di interconnessione tra politica e religione che nella lettura dello studioso trovano un ancoraggio storico grazie ad un'indagine a tutto campo dei legami politici e familiari di tutte le personalità emergenti che condussero al cosiddetto primo triumvirato (pp. 94-98). Questo tipo di analisi ha consentito, infatti, di ipotizzare che la presenza di Clodio ai festeggiamenti riservati alle donne, sia dipesa dalla volontà del futuro tribuno di apparire agli occhi di Pompeia, la moglie di Cesare, come un nuovo Hercules Musarum, non già di fare sesso con lei durante i festeggiamenti. Le simbologie religiose utilizzate dai politici in campo divengono pertanto un punto fermo nella ricostruzione storica dell'episodio che viene così sottratto a incertezze interpretative o a semplificazioni politiche ispirate a palesi partigianerie ${ }^{2}$.

Lungo un percorso di assoluta coerenza con i punti-chiave della monografia trovano spazio i fattori di discontinuità che l'impatto ideologico con il cristianesimo produsse nel pianeta di riti e miti pagani, spazzando via, in prima battuta, l'intricata rete di iniziazioni

2 Sulla questione si veda la ben documentata analisi di L. Fezzi, Il tribuno Clodio, Bari 2008, 34-44. prematrimoniali che attraversavano la "dissacrante" sfera sessuale (vd. p. 13). La rivoluzione "copernicana", che investì il piano etico-sociale, non poteva che penalizzare la complessa miscela di mitologia greco-romana e rituali connessi, svalutando, com'era da aspettarsi, il passaggio verso lo stato matrimoniale, indicato dal dettato evangelico quale linfa vitale della renovanda societas. La rielaborazione dei vecchi schemi sul valore della sessualità dal versante cristiano puntò sull'amore asessuale del dio monoteistico, a fronte della sfrenata attività sessuale degli dèi pagani che guardavano ad amori umani, suscitando interrogativi sull'orizzonte di tutto ciò negli stessi filosofi i quali showed themselves to be much more sophisticated than the common people. D'altronde, anche the pagan writers were often troubled and uneasy when faced with the sexual themes at the core of such ritual and beliefs. They are, therefore, often reticent about embarrassing realities or else merely allude to them (p. 14).

Nel clima di generale cambiamento, al quale concorse la nuova dottrina, autori come Sant'Agostino e polemisti di tutt'altro spessore, gettarono le basi per la secolarizzazione del mondo materiale attraverso la nuova fede, spogliando dei contenuti pagani la vita sessuale, mentre le funzioni derivanti da quella venivano circonfuse di un alone divino (si pensi, per esempio, alle rappresentazioni iconografiche della Madonna che allatta al seno). Mutuazioni e prestiti dal mondo pagano affinarono le tecniche di demonizzazione di divinità come Bona Dea, Giunone, Fauno, Pan, Dioniso, nel nome di una morale trascendente costruita con la duttilità di chi guida il cambiamento, trascurando come i cicli di iniziazione nella Roma pagana tendessero comunque a correggere gli eccessi della sessualità che venivano condannati dalla società stessa.

Questa breve sintesi non rende giustizia della ricchezza e profondità di analisi di Attilio Mastrocinque che, in termini di assoluta originalità ha ricostruito complessi processi culturali in un'area molto vasta del mondo 
antico in cui Bona Dea assolse per secoli funzioni sacre che assorbirono l'interesse di un immaginario collettivo anche al maschile. Né poteva accadere diversamente in una società nella quale anche l'informazione era appannaggio degli uomini e approdo obbligato alla costruzione dei presupposti ideali di uno Stato verticistico. 